\title{
META-ANALYSIS OF THE EFFECTS OF TRANSCRANIAL DIRECT CURRENT STIMULATION ON INHIBITORY CONTROL
}

Philipp A. Schroeder ${ }^{1}$, Tobias Schwippel ${ }^{2}$, Ines Wolz ${ }^{1}$, Jennifer Svaldi ${ }^{1}$

1 Department of Psychology, Clinical Psychology \& Psychotherapy, University of

Tübingen, Schleichstr. 4, 72076 Tübingen, Germany.

2 Department of Psychiatry and Psychotherapy, Neurophysiology \&

Interventional Neuropsychiatry, University of Tübingen, Calwerstr. 14, 72076 Tübingen, Germany

Publication: Schroeder, P. A., Schwippel, T., Wolz, I., \& Svaldi, J. (2020). Meta-Analysis Of The Effects Of Transcranial Direct Current Stimulation On Inhibitory Control. Brain Stimulation, 52(2), 335-344. https://doi.org/10.1249/MSS.0000000000002129

Note. Parts of this research were presented at the $13^{\text {th }}$ International Conference on Complex Medical Engineering (CME) in Dortmund, 2019.

Word count: 3,979 words.

Correspondence and address: Dr. Philipp A. Schroeder; Dept. of Psychology, Clinical Psychology \& Psychotherapy, University of Tübingen, Schleichstr. 4, 72076 Tübingen, Germany; Tel: +49 7071 29-77435, Fax: +49 7071 29-5219, e-mail:

philipp.schroeder@uni-tuebingen.de 


\section{ABSTRACT}

Background: Inhibitory control refers to a central cognitive capacity involved in the interruption and correction of actions. Dysfunctions in these cognitive control processes have been identified as major maintaining mechanisms in a range of mental disorders such as ADHD, binge eating disorder, obesity, and addiction. Improving inhibitory control by transcranial direct current stimulation (tDCS) could ameliorate symptoms in a broad range of mental disorders.

Objective: The primary aim of this pre-registered meta-analysis was to investigate whether inhibitory control can be improved by tDCS in healthy and clinical samples. Additionally, several moderator variables were investigated.

Methods: A comprehensive literature search was performed on PubMed/MEDLINE database, Web of Science, and Scopus. To achieve a homogenous sample, only studies that assessed inhibitory control in the go-/no-go (GNG) or stop-signal task (SST) were included, yielding a total of 75 effect sizes from 45 studies.

Results: Results of the meta-analysis indicate a small but significant overall effect of tDCS on inhibitory control $(g=0.21)$ which was moderated by target and return electrode placement as well as by the task. The small effect size was further reduced after correction for publication bias. 
Conclusion: Based on the studies included, our meta-analytic approach substantiates previously observed differences between brain regions, i.e., involvement of the right inferior frontal gyrus (rIFG) vs. the right dorsolateral prefrontal cortex (rDLPFC) in inhibitory control. Results indicate a small moderating effect of tDCS on inhibitory control in single-session studies and highlight the relevance of technical and behavioral parameters.

Keywords: transcranial direct current stimulation; tDCS; response inhibition; inhibitory control; stop-signal task; go-/no-go task 


\section{INTRODUCTION}

Cognitive control functions enable adaptive behavior in the context of complex environments and cognitive control is broadly corroborated by prefrontal brain networks that can guide attention and influence other neural and motor processes (1-3). Inhibitory control is one important component of cognitive control and refers to the ability to interrupt already initiated or planned motor actions in favor of higher-order goals (4-7). For instance, once the impulse to pick up a candy bar had propelled into the grasping of the highly appetizing energy provider, persons intending to adhere to a non-sugar diet may want to correct this impulse and interrupt the ongoing motor cascade. Accordingly, impairments in inhibitory control have been considered a transdiagnostic mechanism of maintenance across a range of clinical conditions including obesity or binge eating disorder, but also addiction and $\operatorname{ADHD}(3,8,9)$.

In order to improve cognitive control in patients and healthy controls, neurostimulation with transcranial direct current stimulation (tDCS) has been proposed (10-12). By directing a weak direct current through cortical tissue via scalp electrodes, the spontaneous firing activity entailed by a cognitive process can be slightly enhanced or dampened by tDCS, dependent on the targeted brain region and direct current flow (13-16). Changes in behavior can be assessed concurrently ("online"), yet cortico-motor excitability changes will also outlast the stimulation period for up to 1-2 hours dependent on tDCS intensity and duration ("offline" after-effects) $(15,17)$. Performance in cognitive processes can be modulated by application of tDCS dependent on technical parameters (intensity, duration, offline/online, polarity, placement of electrodes) and task parameters 
(task, instruction, stimuli, outcome recordings), which should direct the involvement of a specific neurocognitive function such as inhibitory control (18-21).

Previous quantitative reviews of tDCS studies, however, have documented limited evidence for an effect of prefrontal tDCS on cognitive functions such as inhibitory control in healthy populations (22-24). For instance, Horvath and colleagues (2015) could not document a significant single-session tDCS effect in 59 analyses of different tasks. Moreover, analysis of six studies using the stop-signal task for inhibitory control revealed no significant effect (23). Tremblay and colleagues (2014) reviewed 61 publications and reported high variation across studies (22). In their meta-analysis on cognitive and motor studies, Jacobson and colleagues.(2012) found high variability as well but tDCS polarity effects were present in motor studies only (24).

In general, meta-analyses can suffer from a small size of comparable studies and from the heterogeneity subsumed under a single intervention. In the case of tDCS, one can consider the intrinsic variability provided by tDCS protocols and their non-linear effects, e.g., of electrode and return positioning, size, or intensity $(25,26)$. Moreover, there is also considerable heterogeneity in the assessment of behavior as dependent variable when different tasks are used. Since performance in a task depends on the requirements imposed on individuals, the task features should theoretically affect the recruitment of neural resources as well. When focusing exclusively on executive functions, another more closely confined meta-analysis could document positive evidence if smaller anodes $(<25$ $\mathrm{cm}^{2}$ ) or extracephalic return electrodes were used (20). From a behavioral perspective, still, also this approach subsumed rather heterogeneous tasks - for instance, Imburgio \& Orr (2018) investigated studies with healthy individuals who performed different 
behavioral tasks such as Stroop, Flanker, task-switching, Corsi block, n-back task, and others, which were classified according to Miyake's framework of executive functions (2). Their sub-analysis yielded a descriptive, albeit non-significant effect of anodal tDCS for inhibitory control.

Taken together, previous meta-analyses have interpreted a range of experimental tasks as interchangeable indicators of broad, multifaceted constructs and have - for the most part - ignored the different cognitive mechanisms targeted by each specific task. Against this backdrop, the present meta-analysis focused on two closely related tasks assessing late-control processes: the stop signal task (SST) and the go-/no-go (GNG) task, which both investigate the execution relative to withholding of an already selected or initiated response. Results from behavioral studies indicate that late-control inhibitory processes such as action cancellation and withholding can be separated $(27,28)$ but cancellation triggered stronger fronto-striatal activations (29).

Both GNG and SST assess intentional not-responding in the context of frequent decisions. Next to a likely and timed decision in most trials, the GNG features a particular stimulus for not responding whereas the SST adds an additional stop stimulus to an ongoing motor response after an adaptive delay. Particularly for inhibitory control as assessed in the SST, neurophysiological components have been described as a network incorporating right-lateralized prefrontal regions such as the right inferior frontal gyrus (rIFG), supplementary premotor regions and the motor cortex (30-33). A meta-analysis of fMRI studies utilizing the Go/No-go tasks provided further correlative evidence to a right-lateralized network with particular foci in the pre-supplementary motor area (preSMA) and rIFG, with the former region showing most concurrence for different task types 
(34). Mechanistic studies with transcranial magnetic stimulation have furthered causal contribution of these areas (35). Consequently, a previous tDCS study with experimental variation of the electrode position revealed specific effects for rIFC stimulation compared to right dorsolateral prefrontal cortext (rDLPFC) stimulation (36). Nevertheless, many other stimulation studies also reported enhanced response inhibition from various brain regions including other hubs of the network such as pre-SMA but also co-activated regions such as the prefrontal cortex (PFC), which may be relevant for maintenance of a stopping rule (37). Submitting study results in a meta-analytic approach can further the potential regional selectivity of tDCS on response inhibition in these tasks.

The present meta-analysis compiles single-session tDCS studies on inhibitory control in SST or GNG across healthy controls and clinical populations. Given the large interest into modulations of inhibitory control, we analyze tDCS effects on different brain regions from a relatively high number of 45 publications using SST and GNG tasks only. The synthesized data drawn from qualitatively homogenuous studies allows us to investigate heterogeneity at a more precise level and with adequate number of individual studies (38). We preregistered a series of technical and task parameters to resolve potentially remaining heterogeneity and investigate moderators of a potential tDCS effect on inhibitory control.

\section{METHODS}

The meta-analysis follows the guidelines of the Preferred Reporting Items for Systematic Reviews and Meta Analyses (PRISMA) (39). The study protocol was 
registered before data analysis on PROSPERO on 28 Aug. 2019 and a public version of the registration is available online along with analysis scripts:

\section{https://osf.io/mrxhe/?view only=5bae9f72cf6e498caf9cf02e89297811}

\section{Eligibility criteria}

Only peer-reviewed published studies in English language were included. Studies were required to report experimental designs using active tDCS in human participants with a control condition (sham stimulation, baseline performance, etc.). Only studies implementing the stop-signal task or go-/no-go task and reporting stop-signal reaction time (SSRT) or false alarms (FA), respectively, as outcome from a single-session of tDCS were included. Studies comprised healthy volunteers or clinical samples (i.e., ADHD, fibromyalgia, major depression, obesity, or sub-clinical restrained eating).

\section{Search strategy}

Following the recommendations for reporting systematic reviews (39) two investigators (T.S. and P.A.S.) performed a comprehensive literature search for studies published from data inception to November 2019 including the PubMed/MEDLINE database, Web of Science, and Scopus. The following keywords were used: "transcranial direct current stimulation" OR "tDCS" AND "response inhibition" OR "inhibitory control" OR "stop signal task" OR "go-/no-go task". Further references were manually added from screening the reference sections of retrieved studies, conference contributions, and previous review articles. 


\section{Study selection}

First, all duplicates were removed. Each title and abstract was screened and filtered regarding the relevance for the meta-analytical research question. Relevant publications in English language were reviewed in detail. Cited studies in meta-analyses

or reviews were further included if not detected by the literature search. To obtain comparable effect sizes, we restricted our search on studies using transcranial direct current stimulation (tDCS) and the stop-signal or go-/no-go task; accordingly, studies using different paradigms were excluded (e.g., transcranial magnetic stimulation, transcranial alternating current stimulation, transcranial random noise stimulation, stop anticipation task). All sham-controlled or baseline-controlled experiments comparing effects during or following stimulation with a control condition were included in the present meta-analysis. In this meta-analysis, we focused on single-session experimental studies. However, if the data was reported, the first single-session of training studies was included as well. The study selection process is illustrated in the PRISMA flow chart (Figure 1).

\section{PLEASE INSERT FIGURE 1 HERE}

\section{Tasks and outcome variables}

The effect of interest was the difference between active tDCS condition and control condition in stop-signal reaction time (SSRT) or commission errors / false alarms (FA), as measured by the stop-signal task and the go-/no-go task, respectively. Established procedures for estimation of these outcome measures are available through the literature and are briefly described below. 


\section{Stop Signal Task (SST)}

The stop-signal task investigates action cancellation with stop signals after a certain delay following stimulus presentation for an unrelated go-decision $(4,6)$. The majority of trials (e.g., $75 \%$ ) consist of a go-task and the remaining trials $(25 \%)$ amend a stop-signal after a variable delay (stop-signal delay, SSD) which is continuously adjusted after correct and incorrect inhibition trials. This adaptation procedure yields an overall probability of correct responding in stop trials of $\sim 50 \%$ and thus provides an indirect assessment of the time point when stopping succeeds. The dependent variable of interest in this task is the stop-signal reaction time (SSRT), which is calculated as the difference between response speed in go-trials and mean SSD.

\section{Go-/No-Go Task (GNG)}

In the Go-/No-Go Task, a separate stimulus category requires non-responding (40). The majority of trials (e.g., $75 \%$ ) consist of a go-task and the remaining trials $(25 \%)$ require not-responding based on a predefined rule or context. Thus, the no-go target appears before movement initiation and there is no delay. In the GNG, FA rates are considered as the percentage of no-go trials with uncontrolled answers and failure of inhibitory control.

In both tasks, the dependent outcome is inverse-coded and higher scores in SSRT and FA reflect weaker inhibitory control. Accordingly, negative effect sizes reflect improvements in inhibitory control. 


\section{Data extraction}

The following data were extracted from the included studies:

- Study parameters: parallel or within-subject design, control condition, reporting of blinding success, task (SST vs. GNG), population (healthy controls vs. patients) - Stimulation parameters: tDCS polarity (anodal vs. cathodal electrode over target region) and intensity (range: $0.5 \mathrm{~mA}-2.0 \mathrm{~mA}$ ), electrode size, target and return electrode placement, timing (offline vs. online) - $\quad$ Results: sample sizes, mean and variance of SSRT / FA during real tDCS vs. control condition

\section{Statistical analyses}

Hedges' $\mathrm{g}$ was used as effect size measure and computed to reflect improvements in response inhibition as the standardized mean difference between sham / baseline / control condition and the active tDCS condition. For every active tDCS condition in a study comprising a comparison of active tDCS with control, a single effect size was calculated (e.g., studies comparing two stimulation targets with sham yielded two effect sizes for meta-analysis). If summary statistics for the two conditions were not available in the publication, the authors were contacted or the information was extracted from pictorial displays using WebPlotDigitizer (41). In a small number of five additional publications neither authors nor published materials revealed the data and no effect size could be calculated. 
Meta-regression was performed using the metafor package in $\mathrm{R}$ version 3.5 (42). We first calculated a random effects model to investigate omnibus variance and heterogeneity, which was tested using $Q_{E}$ and quantified using $I^{2}(38)$. The general idea is that the Q-statistic should follow a chi-square distribution under conditions of homogeneity, which can be rejected in case of heterogeneity. Further, the $\mathrm{I}^{2}$-value may capture the extent of heterogeneity. In the present meta-analysis, we expected to follow up on heterogeneity by investigating moderator variables. In a hierarchical approach, the predefined moderator variables (see Table 1) were submitted step-wise and evaluated for significant contribution to the statistical model individually. In each resulting model, we tested whether coefficients for the moderator levels were significantly different (42) and followed-up with separate models for sub-groups in case of significance. A minimum of five studies was required for each sub-group (43), and variables were pooled accordingly (Table 1).

Finally, sensitivity analyses were conducted to investigate the possibility of publication biases. Here, we applied trim-and-fill, PET-PEESE, and detection of outlier studies. In brief, trim-and-fill first removes small studies in order to produce a symmetrical funnel plot with adjusted effect size estimate. In the following filling procedure, the full funnel plot is replicated but missing negative studies are estimated. Trim-and-fill is considered a sensitivity analysis and asymmetry of the funnel plot may indicate that studies are missing (44). PET-PEESE are more recent parametric sensitivity analyses aimed at detecting small-study effects potentially pointing at publication bias $(45,46)$. PET and PEESE are regression-based methods which test for small-study effects and provide corrected effect sizes. Finally, in contrast to the previous methods, the meta-outlier 
detection is diagnostic of highly influential studies who could inflate or deflate metaanalytic results (47). By combining these measures, we sought to increase the robustness of our meta-analytic results.

PLEASE INSERT TABLE 1 HERE

\section{RESULTS}

Study selection led to the inclusion of 45 studies in the meta-analysis, including a total of 75 effect size estimates from 2668 participants. An overview of the descriptive characteristics of all studies is provided in Figure 2 and in supplementary material SM1 ${ }^{1}$.

\section{PLEASE INSERT FIGURE 2 HERE}

\section{Meta-analysis and test of heterogeneity}

The overall effect of tDCS on response inhibition across all studies was small and significant, $g=0.23(\mathrm{SE}=0.046, \mathrm{Cl}[95 \%]=0.14-0.32), \mathrm{z}=5.03, p<.00001$. The model also revealed a low-to-moderate amount of residual heterogeneity, $\mathrm{Q}_{\mathrm{E}}(74)=116.4$, $p=.001, \mathrm{I}^{2}=37.5 \%, \mathrm{CI}[95 \%]=11.8-52.8 \%, \mathrm{AIC}=76.6$, suggesting between-studies variability.

\section{1 https://osf.io/mrxhe/?view only=5bae9f72cf6e498caf9cf02e89297811}




\section{Pre-defined moderators and subgroup analyses}

We specified a minimum of five studies to consider factor levels for moderator analysis (see Table 1), as recommended before (43). The following moderator variables were registered: Study design, control condition, blinding, task, population, tDCS polarity, tDCS intensity, electrode configuration, timing of stimulation. Considering large heterogeneity revealed by qualitative inspection of the included studies, electrode configuration was further aggregated into three factors: target electrode placement, return electrode placement, and electrode size. Following the reviewer suggestions, we also investigated calculated current intensity. In a step-wise procedure, we first tested the significance of each moderator variable individually and respective results of the moderator tests are shown in Table 2.

Entering the three significant moderators task, target placement, and return placement into a single full model led to a slightly reduced amount of residual heterogeneity, $\mathrm{Q}_{\mathrm{E}}(74)=77.8, p=.115, \mathrm{I}^{2}=18.8 \%, \mathrm{Cl}[95 \%]=0.0 \%-44.6 \%, \mathrm{AIC}=75.4$. We next investigated the according sub-groups separately.

\section{PLEASE INSERT TABLE 2 HERE}

\subsection{Effect of task}

Following up on the significant moderator variable test, we fitted separate randomeffects models within each level of task (Figure 2). In total, forty-three comparisons on the stop signal task were evident. The effect of tDCS in the stop signal task was small and significant, $g=-0.33(\mathrm{SE}=0.06), \mathrm{z}=-5.36, p<.0001$. The remaining thirty-two 
comparisons on the go/no-go task revealed no overall effect of tDCS, $g=-0.10$ $(\mathrm{SE}=0.06), \mathrm{z}=-1.66, p=.10$.

\subsection{Effect of target and return electrode placement}

Within each level of the moderator factors, separate random effects models were fitted. Tables 3 and 4 show the results for the individual studies varying in either target or return electrode position.

\section{Sensitivity analyses}

\subsection{Trim and fill}

The trim and fill procedure is a widely recognized sensitivity analysis based on the asymmetry of the funnel plot, which may indicate missing publications (44). If missing values are detected a correction of the effect size can be calculated. Figure 3 illustrates the resulting funnel plot. The regression test for funnel plot asymmetry was significant, $z$ $=-2.71, p=0.007$, and the corrected overall effect size was $d=-.11, z=-2.25, p=.024$. 


\subsection{PET-PEESE}

PET-PEESE are more recent and parametric sensitivity analysis approaches which, in brief, use (squared) standard errors to predict effect size with weighted least squares (meta-regression). Comparable to trim-and-fill, also PET-PEESE cannot completely eliminate bias and the procedures are susceptible to situations with few, small and heterogeneous studies (46). Both PET and PEESE provide a possibility to filter for potential bias, correct effect sizes, and can thus extend the conclusions from other sensitivity analyses.

In this meta-analyses, both tests were significant, PET: $\mathrm{B}=-1.62, p=.007$; PEESE: $B=-2.42, p=.008$, suggesting small-study effects. The corrected overall effect sizes were not statistically significant, gPET $=0.27$, gPEESE $=0.02, z<1.71, p>.15$.

\subsection{Meta-outliers}

Finally, we investigated the presence of outliers according to the method by Viechtbauer and Cheung (2010) in the altmeta package, using metaoutliers() function. According to the analysis, all standardized outliers were smaller than 3 and therefore, no study was declared as outlier.

\section{DISCUSSION}

This meta-analysis investigated the effects of tDCS on inhibitory control in singlesession designs with healthy or clinical populations. Inhibitory control was operationalized by two common behavioral tasks, stop signal task (SST) and go-/no-go task (GNG). Overall, we document a small and significant tDCS effect on response inhibition $(g=0.21)$. 
By collecting data on tightly controlled outcome parameters in two common tasks that require inhibitory control, we additionally investigated modulatory factors, which outlined a significant contribution of target and return electrode placement. Moreover, the effect sizes of tDCS in the SST $(g=0.32)$ was noticeably larger than the effect size of tDCS in the GNG $(g=0.10)$.

The distinct contribution of tDCS to the rIFG in comparison to rDLPFC was investigated in a single design before (36) and our meta-analysis corroborates their results. Precisely, our subgroup analyses indicate a medium effect size for rIFG stimulation whereas rDLPFC stimulation yields an overall null effect. Although the focal specificity of tDCS is considered relatively poor, results still indicate that electrode location matters. Interestingly, we also observed variation due to the placement of the return electrode, which was driven by extracephalic placement compared to various positions across the head. This interesting result is in line with electrode-distance dependent effects on motor-cortical excitability inductions (48) and indicates that the placement of both electrodes influences behavioral outcomes.

The present analyses did not document an effect of tDCS polarity, corroborating previous meta-analytic results that cathodal tDCS may not have substantial effects on cognitive functions (24). Still, the lack of tDCS polarity effects in our results is puzzling given the neurophysiological reduction of motor-cortical excitability expected by cathodal tDCS. Notably, studies in the present meta-analysis often utilized higher current intensities, which may turn excitability-reducing effects of cathodal tDCS into excitabilityenhancing outcomes (49). Interestingly, one study with lower current intensity (0.5 mA) indeed documented the expected reduction of inhibitory control (19). On the other hand, 
only five cathodal tDCS studies targeted the rIFG whereas 21 anodal tDCS studies targeted this region. Of course, ultimately it is the combination of both electrodes through which tDCS elicits its effect and particularly in bilateral electrode settings, placement of the return anode could compensate for the effect of the target cathode, and vice versa. In fact, we saw medium effect sizes for both pre-SMA and rIFG, but null effects, albeit with a magnitude of small effect sizes, for the rDLPFC. Considering the many involved parameters, non-linear interactions involving tDCS intensity, polarity, and electrode placement are possible and the present results may be augmented if core mechanisms are better understood.

Interpretation of the meta-analytic results should consider the outcome of sensitivity analyses, which consistently point at the presence of publication biases. We ran traditional trim-and-fill (44) as well as more recently introduced PET-PEESE analyses $(45,46)$ and observed consistent indications for small-study effects. Nevertheless, the effect-size corrections provided by both methods may underperform in the presence of heterogeneity and should be viewed with caution as all tests suggest missing studies reporting negative results.

Significant heterogeneity was observed in this meta-analysis, still the $I^{2}$ - value of $38 \%$ can be considered small or moderate at best (50). This observation is in line with our strict inclusion criteria only considering results from SST and GNG task, but should be considered for interpretation of the moderator analysis. Potentially homogenous results can be expected from online vs. offline effects during or after stimulation, as excitability changes following anodal stimulation tend to be comparable to online effects $(15,17)$. Interestingly, most of the remaining variance was significantly related to the 
electrode placements and the two tasks which both measure response inhibition behaviorally. We may carefully conclude task variation can significantly shape brain stimulation outcomes at least as much as technical tDCS parameters, as suggested by the proposal of functional specificity (21). Aggregating variation in task parameters could partially explain null results from previous meta-analyses $(20,22-24)$, considering that even the difference between action cancellation and withholding significantly moderated the present meta-analysis.

Since deficits in inhibitory were related to mental disorders such as ADHD, binge eating disorders, obesity, or depression $(3,8,9)$, task-specific targeted improvements are currently being considered as a potential intervention strategy. The present meta-analysis included 12 clinical studies which did not statistically differ from healthy populations. This may point to a potential transfer of the investigated effects to patient groups. However, the present meta-analysis exclusively investigated single-session interventions which usually elicit a brief neuromodulation effect only. So far, the relevant tDCS training studies yielded mixed results. In a recent double-blind training study, patients with alcohol use disorder received anodal tDCS over rIFG during 8-weeks of meditation (51). At baseline and post-intervention, no effects of tDCS vs. sham stimulation on inhibitory control (SSRT) were reported. Moreover, tDCS had no effects on drinking-related outcomes (51). Beyond this, preliminary analyses of an ongoing trial with combined tDCS and an inhibitory control training in children with ADHD showed no effect on clinical symptoms as well (52). These sobering results highlight the difficulty of transferring basic results into clinical trials and the importance of expectancy effects. By contrast, although a study that adopted tDCS over rIFG repetitively over four days in healthy participants (53) observed 
no differences in inhibitory control during the first day of training, the active tDCS group improved on days three and four and performed significantly better than the sham tDCS group. Therefore, these results highlight the present difficulty to examine with confidence which parameters render an effective training across studies and enable a transfer of these effects to clinical symptoms.

A potential limitation of this meta-analysis is that we could not exhaustively model dependent relationships between moderator variables (e.g., tDCS polarity and electrode placement). Moreover, some moderator variable levels were still poorly investigated, e.g., lower tDCS intensities and cathodal stimulation. Due to the standardized task as inclusion criteria, we did not investigate variance in tDCS duration which may affect the overall dose in single- and multiple-session studies. Future studies may also probe differential effects of brain regions in the left hemisphere. A further limitation of our study, as of any meta-analysis, is the dependence on available publications. Since fewer trials used the Go-/No-Go task than the SST, it is possible that relevant parameters were overseen. Moreover, there were too few studies with clinical populations to extract conclusions whether different parameters are effective in patients. On the other hand, the strength of our approach is that we could study a series of potential moderator variables and perform convincing sub-group regression analyses to determine the significance of several factors for modulation of response inhibition.

To conclude, this meta-analysis indicates a small effect of tDCS on inhibitory control. We observed relevant variation in target and return electrode placement in line with a neurocognitive network underlying response inhibition. This result indicates a certain focal sensitivity of different tDCS configurations from a meta-analytic perspective. 
Furthermore, results from stop-signal task revealed higher effect sizes than from go-/nogo task, which highlights the neuromodulatory interactions of tDCS electric fields with functional-behavioral task requirements. Further high-quality studies are needed to investigate potential interactions between technical and functional parameters in tDCS research.

\section{Acknowledgements \& Conflict of Interest}

Work of PS is supported by the German Research Foundation / Deutsche Forschungsgemeinschaft (DFG: SCHR1628/1-1). The authors declare no conflict of interest. 


\section{REFERENCES}

1. Miller EK, Cohen JD. An integrative theory of prefrontal cortex function. Annu Rev Neurosci. 2001 Jan;24:167-202.

2. Miyake A, Friedman NP, Emerson MJ, Witzki AH, Howerter A, Wager T. The unity and diversity of executive functions and their contributions to complex "Frontal Lobe" tasks: a latent variable analysis. Cogn Psychol. 2000;41(1):49-100.

3. Diamond A. Executive Functions. Annu Rev Psychol. 2013;64:135-68.

4. Verbruggen F, Aron A, Band G, Beste C, Bissett P, Brockett AT, et al. Capturing the ability to inhibit actions and impulsive behaviors: A consensus guide to the stopsignal task. Elife. 2019;8:e46323.

5. Wessel JR, Aron AR. It's not too late: The onset of the fron to-central P3 indexes successful response inhibition in the stop-signal paradigm. Psychophysiology. 2016;52(4):472-80.

6. Logan GD, Cowan WB, Davis KA. On the ability to inhibit simple and choice reaction time responses: A model and a method. J Exp Psychol Hum Percept Perform. 1984;10(2):276-91.

7. Newman JP, Widom CS, Nathan S. Passive Avoidance in Syndromes of Disinhibition. Psychopathy and Extraversion. J Pers Soc Psychol. 1985;48(5):1316-27.

8. Hart H, Chantiluke K, Cubillo AI, Smith AB, Simmons A, Brammer MJ, et al. Pattern classification of response inhibition in ADHD: Toward the development of neurobiological markers for ADHD. Hum Brain Mapp. 2014;35(7):3083-94.

9. Lavagnino L, Arnone D, Cao B, Soares JC, Selvaraj S. Inhibitory control in obesity 
and binge eating disorder: A systematic review and meta-analysis of neurocognitive and neuroimaging studies. Neurosci Biobehav Rev. 2016;68:714-26.

10. Brevet-Aeby C, Brunelin J, Iceta S, Padovan C, Poulet E. Prefrontal cortex and impulsivity: Interest of noninvasive brain stimulation. Neurosci Biobehav Rev. 2016;71:112-34.

11. Brunoni AR, Boggio PS, De Raedt R, Benseñor IM, Lotufo PA, Namur V, et al. Cognitive control therapy and transcranial direct current stimulation for depression: a randomized, double-blinded, controlled trial. J Affect Disord. 2014 Jun;162:43-9.

12. Plewnia C, Schroeder PA, Wolkenstein L. Targeting the biased brain: non-invasive brain stimulation to ameliorate cognitive control. The Lancet Psychiatry. 2015 Apr;2(4):351-6.

13. Polanía R, Nitsche MA, Ruff CC. Studying and modifying brain function with noninvasive brain stimulation. Nat Neurosci. 2018;21:174-87.

14. Fertonani A, Miniussi C. Transcranial Electrical Stimulation: What We Know and Do Not Know About Mechanisms. Neurosci. 2017;23(2):109-23.

15. Nitsche MA, Paulus W. Excitability changes induced in the human motor cortex by weak transcranial direct current stimulation. J Physiol. 2000 Sep 15;527 Pt 3(2000):633-9.

16. Priori A, Berardelli A, Rona S, Accornero N, Manfredi M. Polarization of the human motor cortex through the scalp. Neuroreport. 1998 Jul 13;9(10):2257-60.

17. Jamil A, Batsikadze G, Kuo H-I, Labruna L, Hasan A, Paulus W, et al. Systematic evaluation of the impact of stimulation intensity on neuroplastic after-effects induced by transcranial direct current stimulation. J Physiol. 2017;595(4):1273-88. 
18. Jacobson L, Javitt DC, Lavidor M. Activation of Inhibition: Diminishing Impulsive Behavior by Direct Current Stimulation over the Inferior Frontal Gyrus. J Cogn Neurosci. 2011 Nov;23(11):3380-7.

19. Friehs MA, Frings $C$. Cathodal tDCS increases stop-signal reaction time. Cogn Affect Behav Neurosci. 2019;

20. Imburgio MJ, Orr JM. Effects of prefrontal tDCS on executive function: Methodological considerations revealed by meta-analysis. Neuropsychologia. 2018;117:156-66.

21. Bikson M, Rahman A. Origins of specificity during tDCS: anatomical, activityselective, and input-bias mechanisms. Front Hum Neurosci. 2013 Jan;7(October):688.

22. Tremblay S, Lepage J-F, Latulipe-Loiselle A, Fregni F, Pascual-Leone A, Théoret H. The uncertain outcome of prefrontal tDCS. Brain Stimul. 2014 Oct;7(6):773-83.

23. Horvath JC, Forte JD, Carter O. Quantitative review finds no evidence of cognitive effects in healthy populations from single-session transcranial direct current stimulation (tDCS). Brain Stimul. 2015;8(3):535-50.

24. Jacobson L, Koslowsky M, Lavidor M. tDCS polarity effects in motor and cognitive domains: a meta-analytical review. Exp brain Res. 2012 Jan;216(1):1-10.

25. Antal A, Keeser D, Priori A, Padberg F, Nitsche MA. Conceptual and Procedural Shortcomings of the Systematic Review "Evidence That Transcranial Direct Current Stimulation ( tDCS ) Generates Little-to-no Reliable Neurophysiologic Effect Beyond MEP Amplitude Modulation in Healthy Human Subjects: A Systemati. Brain Stimul. 2015;8:27-31. 
26. Schroeder PA, Dresler T, Bahnmueller J, Artemenko C, Cohen Kadosh R, Nuerk H-C. Cognitive Enhancement of Numerical and Arithmetic Capabilities: a MiniReview of Available Transcranial Electric Stimulation Studies. J Cogn Enhanc. 2017 Jan 19;1(1):39-47.

27. Friedman NP, Miyake A. The Relations Among Inhibition and Interference Control Functions: A Latent-Variable Analysis. J Exp Psychol Gen. 2004;133(1):101-35.

28. Stahl C, Voss A, Schmitz F, Nuszbaum M, Tüscher O, Lieb K, et al. Behavioral components of impulsivity. J Exp Psychol Gen. 2014;143(2):850-86.

29. Sebastian A, Pohl MF, Klöppel S, Feige B, Lange T, Stahl C, et al. Disentangling common and specific neural subprocesses of response inhibition. Neuroimage. 2013;64(1):601-15.

30. Banich MT, Depue BE. Recent advances in understanding neural systems that support inhibitory control. Curr Opin Behav Sci. 2015;1:17-22.

31. Sharp DJ, Bonnelle V, De Boissezon X, Beckmann CF, James SG, Patel MC, et al. Distinct frontal systems for response inhibition, attentional capture, and error processing. Proc Natl Acad Sci. 2010;107(13):6106-11.

32. Aron AR, Robbins TW, Poldrack RA. Inhibition and the right inferior frontal cortex. Trends Cogn Sci. 2004;8(4):170-7.

33. Erika-Florence $\mathrm{M}$, Leech $\mathrm{R}$, Hampshire A. A functional network perspective on response inhibition and attentional control. Nat Commun. 2014;5(May):1-12.

34. Simmonds DJ, Pekar JJ, Mostofsky SH. Meta-analysis of Go/No-go tasks demonstrating that fMRI activation associated with response inhibition is taskdependent. Neuropsychologia. 2008;46(1):224-32. 
35. Chambers CD, Garavan H, Bellgrove MA. Insights into the neural basis of response inhibition from cognitive and clinical neuroscience. Neurosci Biobehav Rev. 2009;33(5):631-46.

36. Stramaccia DF, Penolazzi B, Sartori G, Braga M, Mondini S, Galfano G. Assessing the effects of tDCS over a delayed response inhibition task by targeting the right inferior frontal gyrus and right dorsolateral prefrontal cortex. Exp Brain Res. 2015;233(8):2283-90.

37. Hughes ME, Budd TW, Fulham WR, Lancaster S, Woods W, Rossell SL, et al. Sustained brain activation supporting stop-signal task performance. Eur J Neurosci. 2014;39(8):1363-9.

38. Huedo-Medina TB, Sánchez-Meca J, Marín-Martínez F, Botella J. Assessing heterogeneity in meta-analysis: Q statistic or I 2 Index? Psychol Methods. 2006;11(2):193-206.

39. Moher D, Liberati A, Tetzlaff J, Altman DG, and the PRISMA Group. Preferred Reporting Items for Systematic Reviews and Meta-Analyses: The PRISMA Statement (Reprinted from Annals of Internal Medicine). Ann Intern Med. 2009;151(4):264-9.

40. Donders FC. On the speed of mental processes. Acta Psychol (Amst). 1969;

41. Rohatgi A. WebPlotDigitizer. San Francisco, California, USA; 2011. p. 1-23.

42. Viechtbauer W. Conducting meta-analyses in $\mathrm{R}$ with the metafor. J Stat Softw. 2010;36(3):1-48.

43. Van Houwelingen HC, Arends LR, Stijnen T. Advanced methods in meta-analysis: Multivariate approach and meta-regression. Stat Med. 2002;21(4):589-624. 
44. Duval S, Tweedie R. Trim and fill: A simple funnel-plot-based method of testing and adjusting for publication bias in meta-analysis. Biometrics. 2000;56(2):455-63.

45. Moreno SG, Sutton AJ, Thompson JR, Ades AE, Abrams KR, Cooper NJ. A generalized weighting regression-derived meta-analysis estimator robust to smallstudy effects and heterogeneity. Stat Med. 2012;31(14):1407-17.

46. Stanley TD. Limitations of PET-PEESE and Other Meta-Analysis Methods. Soc Psychol Personal Sci. 2017;8(5):581-91.

47. Viechtbauer W, Cheung MW-L. Outlier and influence diagnostics for meta-analysis. Res Synth Methods. 2010;1(2):112-25.

48. Moliadze V, Antal A, Paulus W. Electrode-distance dependent after-effects of transcranial direct and random noise stimulation with extracephalic reference electrodes. Clin Neurophysiol. 2010 Dec;121(12):2165-71.

49. Batsikadze G, Moliadze V, Paulus W, Kuo M-F, Nitsche MA. Partially non-linear stimulation intensity-dependent effects of direct current stimulation on motor cortex excitability in humans. J Physiol. 2013 Apr;591(7):1987-2000.

50. Higgins JPT, Thompson SG. Quantifying heterogeneity in a meta-analysis. Stat Med. 2002;21(11):1539-58.

51. Witkiewitz K, Stein ER, Votaw VR, Wilson AD, Roos CR, Gallegos SJ, et al. Mindfulness-Based Relapse Prevention and Transcranial Direct Current Stimulation to Reduce Heavy Drinking: A Double-Blind Sham-Controlled Randomized Trial. Alcohol Clin Exp Res. 2019;43(6):1296-307.

52. Westwood S, Asherson P, Cohen Kadosh R, Wexler B, Rubia K. A novel neurotherapy of transcranial direct current stimulation (tDCS) combined with 
cognitive training in ADHD children. Brain Stimul. 2019 Mar 1;12(2):521.

53. Ditye T, Jacobson L, Walsh V, Lavidor M. Modulating behavioral inhibition by tDCS combined with cognitive training. Exp brain Res. 2012 Jun;219(3):363-8. 


\section{TABLES}

Table 1. Overview of predefined moderator variables, frequency of effect sizes and pooling of levels in case of few observations.

\begin{tabular}{|c|c|c|c|}
\hline Predefined moderator & level & $n$ & pooled level \\
\hline \multirow[t]{2}{*}{ Study design } & Between-subjects & 25 & \\
\hline & Within-subjects & 50 & \\
\hline \multirow[t]{3}{*}{ Control condition } & Sham tDCS & 63 & \\
\hline & Active control tDCS & 6 & \\
\hline & No tDCS & 6 & \\
\hline \multirow[t]{4}{*}{ Blinding success } & Reported & 19 & a) \\
\hline & $\begin{array}{l}\text { Partially reported } \\
\text { (e.g. no test) }\end{array}$ & 4 & a) Reported \\
\hline & No Blinding & 12 & \\
\hline & Not Reported & 40 & \\
\hline \multirow[t]{2}{*}{ Task } & Stop-Signal Task & 43 & \\
\hline & Go-/No-Go Task & 32 & \\
\hline \multirow[t]{7}{*}{ Population } & Healthy volunteers & 63 & \\
\hline & ADHD adults & 1 & b) \\
\hline & ADHD children & 5 & b) $A D H D$ \\
\hline & Fibromyalgia & 1 & c) \\
\hline & Obesity & 2 & c) \\
\hline & Depression & 2 & c) \\
\hline & Restrained Eater & 1 & c) Other patients \\
\hline \multirow[t]{2}{*}{ tDCS polarity } & Anodal & 53 & \\
\hline & Cathodal & 22 & \\
\hline \multirow[t]{5}{*}{ tDCS intensity } & $0.5 \mathrm{~mA}$ & 2 & d) \\
\hline & $0.7 \mathrm{~mA}$ & 2 & d) \\
\hline & $1 \mathrm{~mA}$ & 22 & d) $\leq 1 \mathrm{~mA}$ \\
\hline & $1.5 \mathrm{~mA}$ & 38 & \\
\hline & $2 \mathrm{~mA}$ & 11 & \\
\hline \multirow{12}{*}{$\begin{array}{l}\text { tDCS current density } \\
(\text { new })^{1}\end{array}$} & $0.028 \mathrm{~mA} / \mathrm{cm}^{2}$ & 14 & \\
\hline & $0.04 \mathrm{~mA} / \mathrm{cm}^{2}$ & 8 & \\
\hline & $0.043 \mathrm{~mA} / \mathrm{cm}^{2}$ & 13 & \\
\hline & $0.055 \mathrm{~mA} / \mathrm{cm}^{2}$ & 2 & e) \\
\hline & $0.057 \mathrm{~mA} / \mathrm{cm}^{2}$ & 5 & e) $0.05 \mathrm{~mA} / \mathrm{cm}^{2}$ \\
\hline & $0.060 \mathrm{~mA} / \mathrm{cm}^{2}$ & 9 & \\
\hline & $0.08 \mathrm{~mA} / \mathrm{cm}^{2}$ & 5 & \\
\hline & $0.094 \mathrm{~mA} / \mathrm{cm}^{2}$ & 11 & \\
\hline & $0.125 \mathrm{~mA} / \mathrm{cm}^{2}$ & 3 & f) \\
\hline & $0.15 \mathrm{~mA} / \mathrm{cm}^{2}$ & 1 & f) \\
\hline & $0.17 \mathrm{~mA} / \mathrm{cm}^{2}$ & 2 & f) \\
\hline & $1.27 \mathrm{~mA} / \mathrm{cm}^{2}$ & 2 & f) $>0.12 \mathrm{~mA} / \mathrm{cm}^{2}$ \\
\hline \multirow{3}{*}{$\begin{array}{l}\text { Target electrode size } \\
(\text { new })^{2}\end{array}$} & $0.79 \mathrm{~cm}^{2}$ & 2 & g) \\
\hline & $9 \mathrm{~cm}^{2}$ & 4 & \\
\hline & $10 \mathrm{~cm}^{2}$ & 1 & g) $\leq 10 \mathrm{~cm}^{2}$ \\
\hline
\end{tabular}




\begin{tabular}{|c|c|c|c|}
\hline & $15.9 \mathrm{~cm}^{2}$ & 2 & h) \\
\hline & $16 \mathrm{~cm}^{2}$ & 12 & h) $\sim 16 \mathrm{~cm}^{2}$ \\
\hline & $19.25 \mathrm{~cm}^{2}$ & 2 & h) \\
\hline & $25 \mathrm{~cm}^{2}$ & 22 & \\
\hline & $35 \mathrm{~cm}^{2}$ & 29 & \\
\hline & not reported & 1 & probably $25 \mathrm{~cm}^{2}$ \\
\hline Target electrode & F3 & 11 & \\
\hline placement & $\mathrm{F} 4$ & 9 & \\
\hline & rIFG & 29 & \\
\hline & $\mathrm{Fz}$ & 4 & i) \\
\hline & $\mathrm{Cz}$ & 2 & i) \\
\hline & $\mathrm{Fp} 1+1 \mathrm{~cm}$ & 2 & i) preSMA \\
\hline & FCZ & 2 & j) \\
\hline & Fp1 & 2 & j) \\
\hline & Fp2 & 2 & j) other frontal \\
\hline & C3 & 2 & k) \\
\hline & Cp3 & 1 & k) \\
\hline & M1 & 2 & k) other central \\
\hline & PPC & 2 & I) \\
\hline & T3 & 3 & I) \\
\hline & Inion & 2 & I) other \\
\hline Return electrode & cheek & 13 & \\
\hline placement & $\begin{array}{l}\text { opposite brain area } \\
\text { (i.e. bilateral tDCS) }\end{array}$ & 20 & m) \\
\hline & vertex & 4 & m) other brain area \\
\hline & supraorbital & 24 & \\
\hline & extracephalic & 14 & \\
\hline Timing & online & 22 & \\
\hline & offline & 53 & \\
\hline
\end{tabular}


Table 2. Tests of moderator variables.

\begin{tabular}{llll}
\hline Moderator variable & $\mathbf{Q}$ & $\mathbf{p}$ & AIC \\
\hline Study design & 0.78 & .68 & 79.5 \\
Control condition & 0.06 & .80 & 78.5 \\
Blinding & 0.60 & .74 & 79.8 \\
Task & 6.92 & $.01^{* *}$ & 72.5 \\
Population & 0.62 & .73 & 79.9 \\
tDCS polarity & 0.63 & .43 & 77.9 \\
tDCS intensity & 0.53 & .77 & 80.0 \\
tDCS current density & 4.96 & .55 & 83.4 \\
Electrode size & 1.54 & .67 & 80.9 \\
Target placement & 23.15 & $.001^{* * *}$ & 70.3 \\
Return placement & 10.06 & $.02^{*}$ & 73.2 \\
Timing & 0.03 & .86 & 78.9 \\
\hline
\end{tabular}

Note. ${ }^{*} p<.05,{ }^{* *} p<.01,{ }^{* * *} p<.001$ 
Table 3. Restricted maximum likelihood (REML) models for transcranial direct current stimulation (tDCS) studies on response inhibition varying in target electrode placement.

\begin{tabular}{lllll}
\hline Target electrode & $\boldsymbol{k}$ & Estimate (SE) & $\mathbf{z}$ & $\boldsymbol{p}$ \\
\hline IDLPFC & 11 & $-0.24(0.10)$ & -2.22 & .026 \\
rDLPFC & 9 & $0.13(0.11)$ & 1.19 & .234 \\
rIFG & 29 & $-0.39(0.06)$ & -6.12 & .0001 \\
preSMA & 8 & $-0.39(0.17)$ & -2.30 & .021 \\
other frontal & 6 & $-0.23(0.14)$ & -1.62 & .105 \\
other central & 5 & $-0.27(0.15)$ & -1.85 & .065 \\
other & 7 & $-0.11(0.12)$ & -0.91 & .363 \\
\hline
\end{tabular}

Table note. IDLPFC = left dorsolateral prefrontal cortex, rDLPFC = right dorsolateral prefrontal cortex, $\mathrm{rIFG}=$ right inferior frontal gyrus; preSMA = pre-supplementary motor area. Other frontal areas: orbitofrontal cortex (4), medial frontal cortex (2). Other central areas: primary sensory cortex (1), primary motor cortex M1 (4). Other areas: right posterior parietal cortex (2), temporo-parietal cortex (3), cerebellum (1), occipital cortex (1). 
Table 4. Restricted maximum likelihood (REML) models for transcranial direct current stimulation (tDCS) studies on response inhibition varying in return electrode placement.

\begin{tabular}{lllll}
\hline Return electrode & $\boldsymbol{k}$ & Estimate (SE) & $\mathbf{z}$ & $\boldsymbol{p}$ \\
\hline cheek & 13 & $-0.43(0.10)$ & -4.31 & .0001 \\
other brain area & 24 & $-0.22(0.08)$ & -2.86 & .004 \\
Supraorbital & 24 & $-0.30(0.08)$ & -3.74 & .0002 \\
Extracephalic & 14 & $-0.01(0.11)$ & -0.09 & .92 \\
\hline
\end{tabular}




\section{FIGURES}

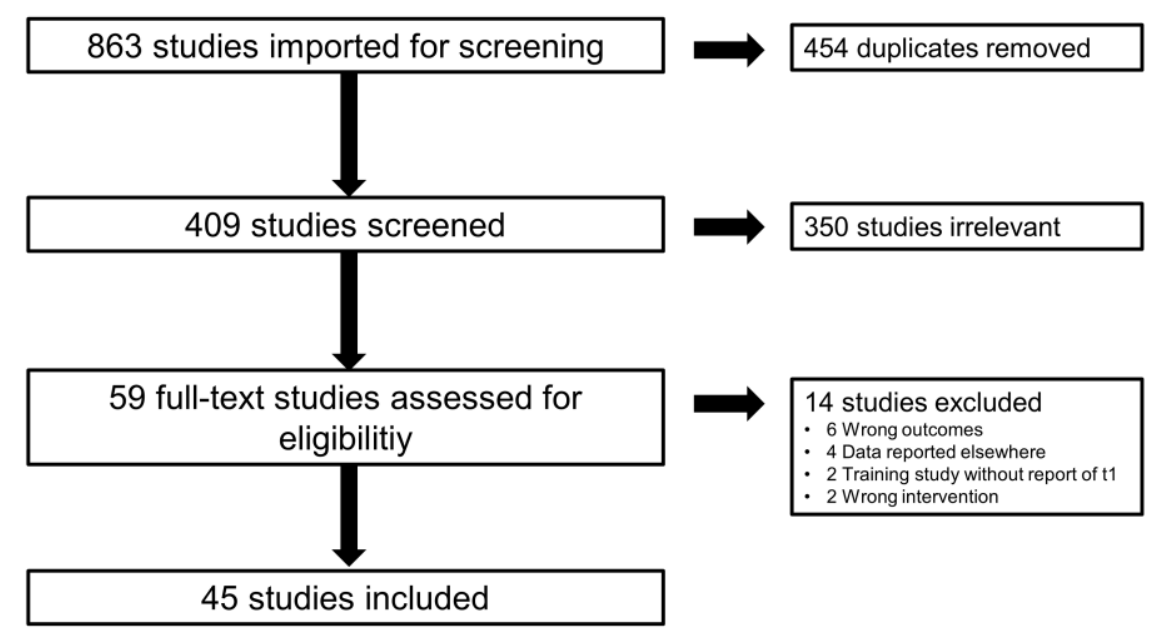

Figure 1. PRISMA flow chart of literature search and inclusion of articles. 
$\sin$

Go-/No-Go Task

Nejati (2017) Exp. 2 \#2 Boggio (2007) \#2

Soltaninejad (2019) \#2

Nejati (2018) \#1

Boggio (2007) \#1

Dambacher (2015) \#2

Nejati (2017) Exp. 2 \#

Nieratschker (2015

Wynn (2019)

Friedrich \& Beste (2018)

Nejati (2018) \#2

Dambacher (2015) \#1
Molero-Chamizo (2018)

Sikström (2016)

Nejati (2017) Exp. 1

Campanella (2018)

Leite (2018) \#1

Soltaninejad (2019) \#1

Adelhofer (2019)

Lapenta (2012) \#

Cosmo (2015)

Lapenta (2012) \#2

Silva (2017)

Leite (2018) \#2

Sallard (2018)

Beeli (2008) \#1

Boggio (2008)

Osimo (2019) \#2

Sedgmond (2019)

Osimo (2019) \#1

Plewnia (2014)

Summary Effect Šize GNo

Stop Signal Task

Yu (2015) Exp. 1

Kwon (2013) \#2

Jacobson (2011) \#1

Chen (2019)

Cai $(2016) \# 1$

Sandrini (2019)

Castro-Meneses (2016) \#2

Castro-Meneses (2016) \#1

Friehs \& Frings (2018)

Hogeveen (2016) \#2

Jacobson (2011) \#3

Hogeveen (2016) \#1

Stramaccia (2015) \#3

Cunillera (2014)

Hsu (2011) \#1

Li (2019) \#1

Li (2019) \#2

Stramaccia (2015) \#4

Hsu (2011) \#2

Jacobson (2011) \#4

Stramaccia (2017) \#2

Mansouri (2017)

Ouellet (2015) \#1

Kwon (2013) \#1

Cai (2016) \#2

Liang (2014)

Reinhart \& Woodman (2014)

Hsu (2011) \#4

Hsu (2011) \#3

Ditye (2012) Session 1

Jacobson (2011) \#

Ouellet (2015) \#2

Stramaccia (2015) \#2

Bender (2017) Exp. 2 \#2

Stramaccia (2015) \#1

Stramaccia (2017)\#1

Hogeveen (2016) \#4

Cunillera (2016)

Hogeveen (2016) \#3

Reinhart \& Woodman (2014)

Jacobson (2011) \#5

Friehs \& Frings (2019)

Summary Effect šize šs

Summary Effect Size Total (REML)

N

(10)

\begin{tabular}{ll|l}
$10 \longmapsto$ \\
7 \\
20
\end{tabular}

$-0.83[-1.93,0.26]$

$-0.71[-1.30,-0.13]$

$-0.65[-1.60,0.31]$

$-0.63[-1.25,-0.01]$

$-0.59[-1.49,0.30]$

$-0.36[-0.80,0.08]$

$-0.36[-0.91,0.19]$

$-0.35[-1.02,0.33]$

$-0.33[-0.90,0.24]$

$-0.29[-0.90,0.32]$

$-0.19[-1.07,0.69]$

$-0.15[-0.77,0.47]$

$-0.11[-0.83,0.60]$

$-0.11[-0.81,0.60]$

$-0.06[-0.75,0.64]$

$-0.05[-0.67,0.57]$

$-0.05[-0.69,0.59]$

$-0.05[-0.57,0.48]$

$-0.00[-0.51,0.50]$

$0.02[-0.50,0.55]$

$0.05[-0.42,0.52]$

$0.06[-0.63,0.75]$

$0.10[-0.60,0.79]$

$0.12[-0.35,0.58]$

$0.12[-0.62,0.86]$

$0.15[-0.24,0.54]$

$0.17[-0.13,0.47]$

$0.18[-0.21,0.57]$

$0.20[-0.21,0.61]$

$0.10[0.210 .02]$
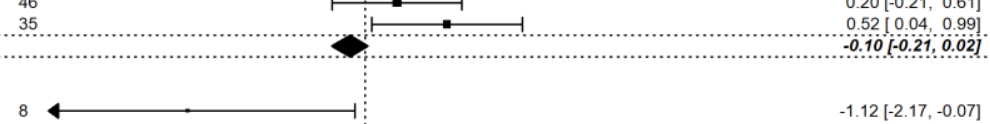

$-1.12[-2.17,-0.07]$

$-1.03[-1.49,-0.56]$

$-0.88[-1.76,-0.01]$

$-0.83[-1.37,-0.29]$

$-0.81[-1.42,-0.19]$

$-0.81[-1.55,-0.06]$

$-0.68[-1.44,0.08]$

$-0.67[-1.43,0.09]$

$-0.66[-1.20,-0.13]$

$-0.65[-1.37,0.07]$

$-0.64[-1.50,0.21]$

$-0.64[-1.36,0.08]$

$-0.60[-1.16,-0.04]$

$-0.59[-1.20,0.01]$

$-0.57[-1.32,0.19]$

$-0.56[-1.14,0.02]$

$-0.49[-1.06,0.09]$

$-0.43[-0.99,0.12]$

$-0.40[-1.15,0.35]$

$-0.39[-1.23,0.45]$

$-0.37[-0.95,0.21]$

$-0.37[-0.94,0.20]$

$-0.37[-1.09,0.35]$

$-0.34[-0.78,0.10]$

$-0.33[-0.92,0.27]$

$-0.32[-0.98,0.34]$

$-0.19[-0.84,0.47]$

$-0.16[-0.90,0.58]$

$-0.13[-0.87,0.61]$

$-0.12[-0.96,0.72]$

$-0.10[-0.94,0.73]$

$-0.10[-0.82,0.62]$

$-0.09[-0.64,0.46]$

$-0.08[-0.73,0.57]$

$0.00[-0.54,0.55]$

$0.03[-0.54,0.59]$

$0.03[-0.68,0.73]$

$0.20[-0.57,0.97]$

$0.23[-0.48,0.93]$

$0.23[-0.42,0.89]$

$0.26[-0.39,0.92]$

$0.54[-0.31,1.39]$

$-0.23[-0.32,-0.14]$

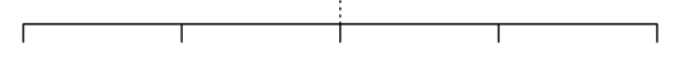

Summary effect sizes for single-session transcranial direct current stimulation (tDCS) effect

on response inhibition (active tDCS vs. control condition) in Go-No-Go and Stop Signal Tasks 
Figure 2. Summary effect sizes for single-session transcranial direct current stimulation (tDCS) effect on response inhibition (active tDCS vs. control condition) in Go-No-Go and Stop Signal Tasks. $\mathrm{N}$ refers to total sample size in within-subject designs and to active group size in between-subject designs.

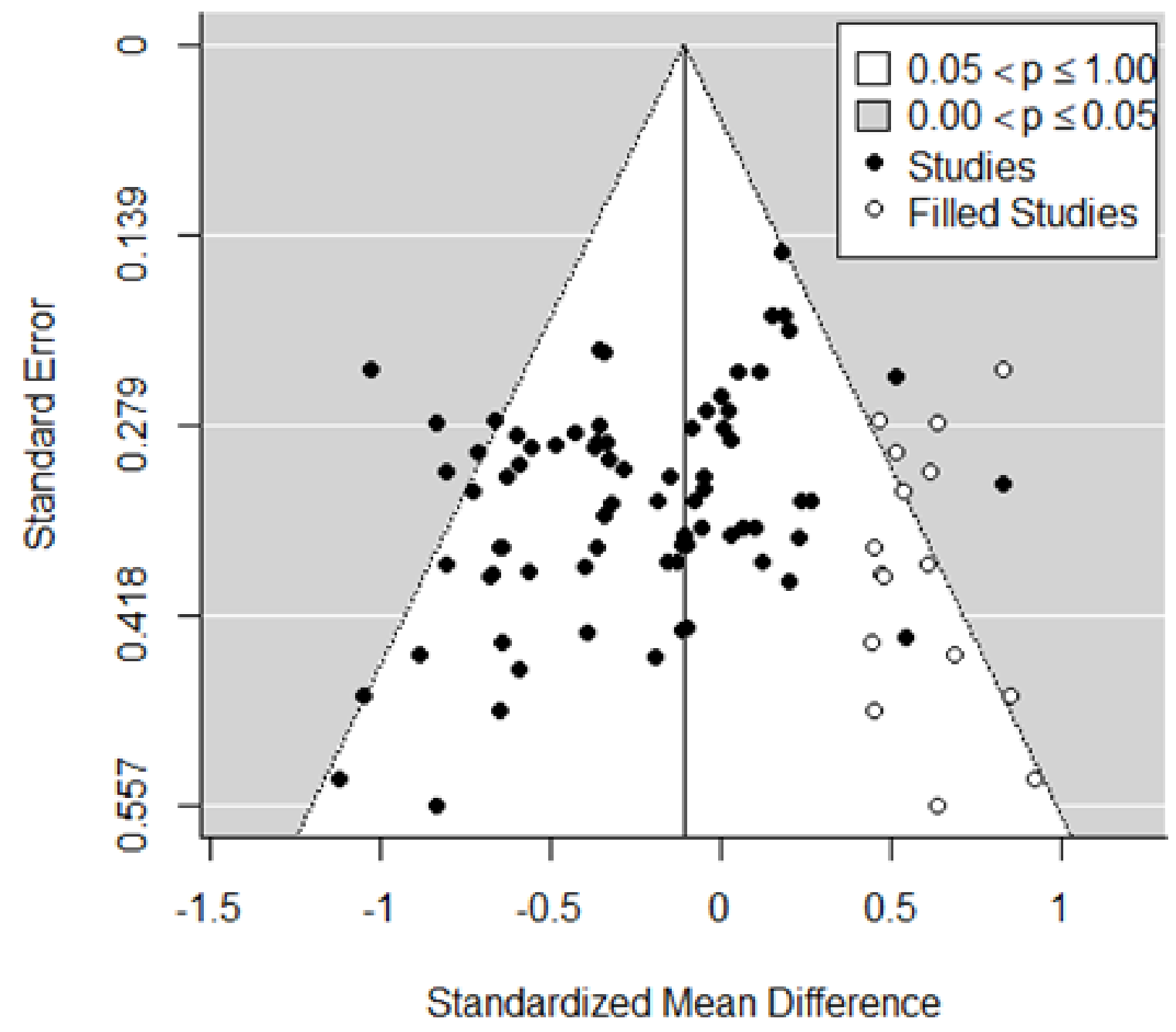

Figure 3. Funnel plot resulting from trim-and-fill procedure. 